\title{
To evaluate the use of surgical safety checklist in a tertiary referral obstetrics center of Northern India
}

\begin{abstract}
Background: Surgical related morbidities and mortalities has been reported to reduce significantly after the introduction of surgical safety checklist (SSCL).Its use in obstetrics is more challenging due to greater number of high risk emergency cases. Obstetrical patients need consideration for mother as well as for the foetus and omission of any step may result in significant adverse events. Despite the introduction of surgical safety checklist in our institute, compliance is not as good as in gynaecological theatres, therefore we undertook this pilot study to evaluate the use of surgical safety check list in the obstetrical theatre of our institute and analyse it in terms of staff attitude, compliance and patient effects.
\end{abstract}

Methodology : This study was a prospective evaluation of study population of thirty people comprising theatre nurses, anaesthetists, paediatricians and obstetricians over a period of three and half month. Staff attitude to the use of modified SSCL and various barriers was assessed with the help of semi-structured questionnaire.

Results: The compliance to the use of SSCL increased during the study period to upto $40 \%$. The overall attitudes towards use of SSCL was positive $(>96 \%)$ and the main barriers to the use of SSCL turned out to be lack of time and lack of assertiveness of staff (>80\%).

Conclusion: Even an overwhelmingly positive attitudes by staff does not ensure compliance There is a need to address sociocultural issues in an individual set up, e.g workload of particular team members, support from seniors and timing of carrying out SSCL etc.
Volume 9 Issue 2 - 2018

\author{
Neelam Aggarwal,' Navneet Dhaliwal,'² Bharti \\ Joshi' \\ 'Department of Obstetrics \& Gynecology, PGIMER Chandigarh, \\ India \\ ${ }^{2}$ Department of hospital administration, PGIMER Chandigarh, \\ India
}

Correspondence: Dr. Bharti Joshi, Assistant Professor (MBBS, MD, DNB), Department of Obstetrics \& Gynecology, Post Graduate Institute of Medical Education and Research Chandigarh, India, Tel 919915166210,

Email drbhartijoshi09@gmail.com

Received: November 25, 2017 | Published: April II, 2018

Keywords: surgical safety checklist, compliance, obstetrics, attitude

\section{Abbreviations: SSCL, Surgical safety checklist}

\section{Background}

Surgical care is an essential component of global health care but not without adverse events. Major complications rates in surgical procedures are reported to be $3-16 \%$ with death rate of 0.4 to $2.7 \%$ in well-resourced countries. Complications are much higher in resource limited settings with mortality rate upto $5-10 \%$ from major surgeries. ${ }^{1-3}$

Introduction of a peri-operative surgical safety checklist (SSCL) by WHO has been reported to significantly reduce the surgical morbidity and mortality. There has been found significant reduction in mortality to almost one half and morbidity by one third with the use of surgical safety checklist. ${ }^{4-7}$ Use of surgical safety checklist worldwide has been evaluated and reported in various studies. ${ }^{1-4,7-9}$ Surgical safety checklist can be of great utility in obstetrics but compliance to the use of such checklist is more challenging compared to the elective gynaecological surgeries. The reason may be the higher number of high risk emergency obstetrical cases .In obstetrical theatre multidisciplinary team consisting of obstetricians, anaesthetists and neonatologists usually work together and thus presents a number of challenges as it requires a good leadership for successful team work .Obstetrical patients need consideration for mother as well as for the foetus and omission of any step may result in significant adverse events. In our institute, a tertiary referral centre the surgical safety checklist has been introduced since one year in both obstetrical and gynaecological operation theatres. But we feel that compliance is not as good as in gynaecological theatres. Since there have been no studies from India; We undertook this as pilot to evaluate the use of surgical safety check list in the obstetrical theatre of our institute and analyse it in terms of staff attitude, compliance and patient effects.

\section{Methodology}

This study was a prospective evaluation of study population of thirty people carried in the obstetrical theatre of apex institute of Northern India. Study population included thirty people comprising theatre nurses, anaesthetists, paediatricians and obstetricians. All the surgeries carried out during study period were recorded. This study aimed to assess the attitude of the staff regarding the use and benefits of SSCL. Surgical cases performed in the labour room operation theatre were also analysed.

\section{Study period}

Study period is $1 / 2 / 2015-15 / 5 / 2015$

\section{Data collection techniques}

a. Primary data: Staff attitude to use of SSCL, Barriers in the procedure

b. Secondary data: Record of all surgeries in the labour room theatre during a study period.

\section{Tool making}

Questionnaire: For assessment of staff attitude and barriers to use of SSCL (Annexure I). A semi structured questionnaire was prepared and distributed among thirty people (study population) including theatre nurses, anaesthetist, paediatrician and obstetrician. This questionnaire was intended to access the attitude of the staff to use of surgical safety checklist (SSCL) and also barriers to its use. All the 
concerned personnel were instructed to use SSCL in each use .The same questionnaire (as in pre-test) was again distributed at the end of the study to examine the staff attitude and analyse the barriers to its use.

\section{Attitudes to Surgical Checklist Questionnaire}

\begin{tabular}{|c|c|c|c|c|c|c|}
\hline S. No & & Disagree strongly Disagree slightly & Neutral & Agree slightly & Agree slightly & Do not know \\
\hline I & $\begin{array}{l}\text { The complete checklist is used for } \\
\text { every cesarean in CLR-OT }\end{array}$ & 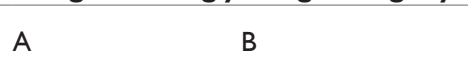 & C & D & $\mathrm{E}$ & DK \\
\hline 2 & $\begin{array}{l}\text { Sometimes sections of the checklist } \\
\text { are not completed }\end{array}$ & A & C & $\mathrm{D}$ & E & DK \\
\hline 3 & $\begin{array}{l}\text { I believe using the checklist reduces } \\
\text { the likelihood of human error }\end{array}$ & A & C & $\mathrm{D}$ & E & DK \\
\hline 4 & $\begin{array}{l}\text { I believe using the checklist improves } \\
\text { patient safety }\end{array}$ & A & C & $\mathrm{D}$ & $\mathrm{E}$ & DK \\
\hline 5 & $\begin{array}{l}\text { I believe using the checklist improves } \\
\text { interspeciality in theatre }\end{array}$ & A & C & $\mathrm{D}$ & $\mathrm{E}$ & DK \\
\hline 6 & $\begin{array}{l}\text { The use of the checklist should be } \\
\text { mandatory for ever case }\end{array}$ & A & C & $\mathrm{D}$ & E & DK \\
\hline 7 & $\begin{array}{l}\text { I intend to initiate the use of the } \\
\text { checklist in the future. }\end{array}$ & A & C & D & $E$ & DK \\
\hline
\end{tabular}

\section{Barriers to the use of Surgical Checklist}

\begin{tabular}{lllllll}
\hline S. No & & Disagree strongly Disagree slightly & Neutral & Agree slightly & Agree Slightly & Do not Know \\
\hline I & Lack of awareness & A & B & C & D & DK \\
2 & Lack of assertiveness to staff & A & B & C & D & E \\
3 & Lack of time & A & B & C & D & D \\
4 & lack of training & A & B & C & D & DK \\
5 & Lack of attitude & A & B & C & D & DK \\
\hline
\end{tabular}

Surgical safety checklist (SSCL): W.H.O surgical safety checklist was modified to our local needs and this SSCL for labour room was validated by senior obstetric consultants of the department and then

\section{Step I-Before induction of anesthesia} Nurse

Whether following points have been confirmed

a. Patient safety

b. Type of surgery

Obstetrical

Non- obstetrical

e.Procedure

f. Written consent

g. Whether resuscitation fluids available (blood,

crystalloids, colloids) Y N

\section{Anesthetist}

Whether following points have been checked.

a. Anesthesia machine and drugs

b. Preoperative monitors Functioning and attached ECG PULSE OXIMETER NIBP

c. Has the indication and amount of blood loss certified by the obstetrical team. $\mathrm{YN}$

\section{Surgeon}

a. Indication

b. Risk of blood loss of $10 \%$ or more of the estimated blood volume? Y/N

If yes, is IV access, central line, fluids planned.

c.Need of interdepartmental assistance $\mathrm{Y} N$

\section{Pediatrics}

a. Has the person arrived

b. Indication and gestation known clearly finalized. All the surgeries then analysed regarding use of SSCL (Annexure II).

\author{
Step 2-Before the skin incision \\ Surgeon, anesthesia and nurse \\ The team confirms: \\ a. Patient's name \\ c. Procedure \\ d.Availability of blood
}

\section{Nurse}

a. Has the sterility been confirmed $\mathrm{Y} N$

b. Are there any equipment issues or any concerns. $\mathrm{Y} N$

\section{Anesthetist}

a. Sensitivity to prophylactic antibiotic checked. Y N

b. Has the antibiotic prophylaxis been given within last 30 minutes $Y N$

c. Are there any patient specific concerns?
Step 3-Before patient leaves OT Nurse confirms

a.Count of instruments, sponges, cotton b.Check oxytocin drip

Surgeon, anesthesits and nurse Specific post-operative recovery instructions for the team 
An independent worker interviewed the concerned staff to assess the attitudes and barrier to the use of SSCL. After that the residents were instructed to use it in every surgical procedure. The circulating staffs were told to remind the resident about each case.

All the surgical details of the cases performed from 1/2/2015 to $15 / 5 / 2015$ were recorded in details regarding nature, indication, details of the procedure and compliance to the use of SSCL. The data was recorded in an excel sheet later on analysis.

Descriptive analysis was done to describe the types of surgeries and variables were compared with the help of chi square test. A p value $<0.05$ was taken as significant.

\section{Results}

A total of 501 surgeries were performed during the study period, out of these $402(81.5 \%)$ were emergency surgeries and rest were elective $(18.5 \%)$ (Table 1$)$. Out of these total surgeries additional procedures were required to be carried out due to intraoperative complications. These procedures were peripartum hysterectomy, internal iliac ligation and bakri ballon insertion. This emphasizes the load of emergency burden in the labour room and high stress on clinicians as well as anaesthetists and neonatologist.

Table I Distribution of surgeries

\begin{tabular}{lllll}
\hline Month & $\begin{array}{l}\text { Emergency } \\
\text { LSCS -N (\%) }\end{array}$ & $\begin{array}{l}\text { Elective } \\
\text { LSCS N (\%) }\end{array}$ & Cervical cerclage & Total \\
\hline February & $97(82.9 \%)$ & $16(13.6 \%)$ & 4 & 117 \\
March & $128(82.05 \%)$ & $27(17.3 \%)$ & $\mathrm{I}$ & 156 \\
April & $115(77.7 \%)$ & $30(20.2 \%)$ & 3 & 148 \\
May & $62(77.5 \%)$ & $18(22.5 \%)$ & 0 & 80 \\
\hline
\end{tabular}

The compliance to the use of SSCL was checked in each case from March to May 15th, 2015. It is clear from the Table 2 that use of SSCL compliance increased during the study period to upto $40 \%$. Even though the use has increased but it is far less than expected.

Table 2 Compliance to the use of SSCL

\begin{tabular}{lllll}
\hline Month & Compliance N \% & Emergency & Elective & P value \\
\hline February & $0 / 113(0 \%)$ & 0 & & Nil \\
March & $49 / 155(31.6 \%)$ & 40 & 9 & $<0.05 \% *$ \\
April & $54 / 145(37.2 \%)$ & 40 & 14 & $<0.05 \%^{*}$ \\
May & $32 / 80(40 \%)$ & 26 & 6 & $<0.05 \% *$ \\
\hline
\end{tabular}

$* \mathrm{P}<0.05 \%$

\section{Staff attitude}

The questionnaire was distributed among 30 personnel including nurses, technicians and clinicians including obstetricians, anaesthetists and paediatricians .Most of them were aware of the SSCL and also agreed upon the benefits of its use especially inter-professional communication. They were also aware of incomplete and inconsistent use of SSCL even though they believed in making use of SSCL mandatory but were not sure as to who should lead the initiation of SSCL and take responsibility of completing it. The overall attitudes towards SSCL was positive $(>96 \%)$.

\section{Barriers}

Main barriers to the use of SSCL turned out to be lack of time and lack of assertiveness of staff $(>80 \%)$.Especially in our set up ,where cases are high risk and report in emergency, residents are busy in sorting out multiple other aspects that they do not comply with use of SSCL even though their attitudes are positive.

\section{Discussion}

The purpose of index study was to evaluate the use of surgical safety checklist in obstetrical theatre of our institute and to obtain information on attitudes to the use of SSCL. The SSCL was introduced in labour room theatre two years ago but its use had fallen to almost nil presently. A particular area worth highlighting is the amount of emergency surgeries in this theatre $(>80 \%)$. Although the compliance improved to only $40 \%$ during the study period regarding the use of SSCL in every surgical case; but the overall attitude of the staff towards SSCL was overwhelmingly positive.

This finding of positive attitude is consistent with other studies examining attitudes towards SSCL in literature. ${ }^{2,7,8}$ Nurses were found to be particularly positively disposed to the checklists as compared to other personnel. ${ }^{9}$ But in our study, the junior residents were responsible for initiating the SSCL. In our study, lack of time and assertiveness was found to be the main barrier as residents responsible for initiating the SSCL.

Various barriers have been found to the use of the check list in different studies reported in literature .In our study, lack of time and lack of assertiveness was found to be the main barrier as residents responsible for initiating the SSCL use were also in other things like arranging the blood drugs , I/V fluids getting consent etc. Also high risk due to our hospital being tertiary referral center demanded immediate attention to their associated problems. Other studies have reported steep hierarchy of surgical team as a barrier to nurses being check coordinators. ${ }^{9}$ O'Connor et al. ${ }^{8}$ in their study reported barriers as need of getting their signatures, poor team work and the timing of carrying out checklist. The timing is a particular problem for the anaesthetists as checklists tends to be initiated during the periods of high workload prior to surgery. ${ }^{9}$

Even though our study period was limited it does bring out some important points. As majority of the surgeries in one labor room operation theatre are emergency procedures, SSCL would prove to improve the inter-professional communication and patient safety. All members of the theatre team must be involved in the checklist procedure.

Giving the job of initiating the checklist to the circulating nurse might improve the compliance as residents in their busy schedule may not be able to complete it. However, senior members must support the use SSCL and insist upon its use. There is a need for continued reinforcement of SSCL use for compliance as it is clear from our own experience where use almost dwindled to nil with time. Similar variation in compliance has been reported by Vats et al. ${ }^{9}$ from UK where research team needed to be constantly present to encourage compliance. Low compliance as seen in our set up has also been explained by Yuan et al., ${ }^{10}$ who suggests that checklist's mechanism of improvement is likely to be influenced by availability of resources needed to complete the recommended process. Apart from our own experience, the recommendation for improving compliance to the use of SSCL from broader literature also include involvement of all team members, support from senior members, ongoing education and training and addressing barriers in an individual set up.

\section{Conclusion}

The surgical safety checklist is potentially very effective system for avoiding many potential adverse events in surgery .However, even 
overwhelmingly positive attitudes by staff does not ensure compliance .There is a need to address sociocultural issues in an individual set up, e.g workload of particular team members, support from seniors and timing of carrying out SSCL etc. In our set up the responsibility of initiation of SSCL seems better with circulating nurse as resident has too many clinical issues at hand. Moreover, it should be made interdisciplinary for best outcomes.

\section{Acknowledgements}

None.

\section{Conflicts of interest}

Authors declare there is no conflict of interest in publishing the article.

\section{References}

1. Singh SS, Mehra N, Hopkins L. Surgical Safety Checklist in Obstetrics and Gynaecology. J Obstet Gynaecol Can. 2013;35(1)82-83.

2. Haynes AB, Weiser TG, Berry WR, et al. A surgical safety checklist to reduce morbidity and mortality in a global population. $N$ Eng $J$ Med. 2009;360(5):491-499.
3. De Vries EN, Ramrattan MA, Smorenberg SM, et al. The incidence and nature of in-hospital adverse events: systemic review. Qual Saf Health Care. 2008;17(3):216-23.

4. Burbos N, Morris E. Applying the World Health Organisation Surgical Safety Checklist to obstetrics and gynaecology. Obstetrics, Gynaecology \& reproductive Medicine. 2011;21(1):24-26.

5. RCOG statement on the NPSA surgical checklist for maternity. London: The Royal College of Obstetrician and gynaecologists; 2011.

6. American College of Obstetricians and Gynaecologists. Patient safety in the surgical environment. Committee Opinion No.464. 2010;116:786-90.

7. Nilsson L, Lindberget $\mathrm{O}$, Gupta A, et al. Implementing a pre-operative checklist to increase patient safety: a 1-year follow-up of personnel attitudes. Acta Anaesthesiol Scand. 2010;54(2):176-182.

8. O Connor P, Reddin C, O Sullivan M, et al. Surgical checklists: the human factor. Patient Saf Surg. 2013;(1)7:14

9. Vats A, Vincent CA, Nagpal K, et al. Practical challenges of introducing WHO surgical checklist: UK pilot experience. BMJ. 2010;340:b5433.

10. Yuan CT, Walsh D, Tomarken JL, et al. Incorporating WHO Surgical Safety Checklist Practieat two hospitals in Liberia. Jt Comm J Qual Patient Saf. 2012;38(6):254-260. 